\title{
The IncRNA myocardial infarction associated transcript-centric competing endogenous RNA network in non-small-cell lung cancer
}

This article was published in the following Dove Press journal:

Cancer Management and Research

\author{
Chang Zheng ${ }^{1,2}$ \\ Xuelian $\mathrm{Li}^{2}$ \\ Biyun Qian ${ }^{3}$ \\ Nannan Feng ${ }^{3}$ \\ Sumeng $\mathrm{Gao}^{3}$ \\ Yuxia Zhao ${ }^{4}$ \\ Baosen Zhou ${ }^{1,2}$ \\ 'Department of Clinical Epidemiology \\ and Center of Evidence-Based \\ Medicine, The First Affiliated Hospital, \\ China Medical University, Shenyang \\ I I000 I, People's Republic of China; \\ ${ }^{2}$ Department of Epidemiology, School \\ of Public Health, China Medical \\ University, Shenyang II0I22, People's \\ Republic of China; ${ }^{3}$ Department of \\ Epidemiology, School of Public Health, \\ Shanghai Jiao Tong University, Shanghai \\ 200240, People's Republic of China; \\ ${ }^{4}$ Department of Radiotherapy, The \\ Fourth Affiliated Hospital of China \\ Medical University, Shenyang, II0032, \\ People's Republic of China
}

Correspondence: Baosen Zhou Department of Clinical Epidemiology and Center of Evidence-Based Medicine, The First Affiliated Hospital, China Medical University, No. 155 Nanjing Bei Street, Shenyang, Liaoning I I000I, People's Republic of China

Tel +86 I338688 I563

Email bszhou@mail.cmu.edu.cn
Background: The leading cause of death for cancer is lung cancer, of which the majority subtype is non-small cell lung cancer (NSCLC). Recent studies have shown long non-coding RNAs are transcribed and contribute to cancer. Previous study has shown that a few single nucleotide polymorphisms (SNPs) in myocardial infarction associated transcript (MIAT) were associated with some diseases or function as competing endogenous RNA (ceRNA) in some cancer.

Patients and methods: We performed bioinformatic methods for analyzing RNA-seq and miRNA-seq data of NSCLC from The Cancer Genome Atlas database. 1352 NSCLC patients and 1320 cancer-free controls for genotyping, and dual luciferase reporter assay, real-time PCR are performed in A549 and H1975 lung cancer cell lines. Results are analyzed by SPSS v16.0.

Results: In the present study, we focus on the role of over-expression MIAT in NSCLC. We confirmed that rs1061451 $\mathrm{T}>\mathrm{C}$ (allele odds ratio $=0.22 ; P<0.01$ ) was associated with NSCLC. Furthermore, we constructed MIAT-centric ceRNA network, and three mRNAs (MYO1B, SGK1 and $W N T 9 A$ ) was identified as targets by MIAT via miR-133a-5p.

Conclusion: C-containing genotypes of MIAT rs1061451 were protective factor of NSCLC, and MIAT, which may act as ceRNA via miR-133a-5p, modulated MYO1B, SGK1 and WNT9A expression level.

Keywords: non-coding RNA, non-small-cell lung cancer, single-nucleotide polymorphism, competing endogenous RNA

\section{Introduction}

Thanks to Genomic Projects, it is evident that more than three quarters of the human genome is actively transcribed as non-coding RNAs (ncRNAs), ${ }^{1}$ which play major biological roles in the regulation of RNA expression by influencing cellular development and other functions. ${ }^{2}$ Based on the transcript size, ncRNAs can be grouped into microRNA (miRNA, 20-23 nucleotides) and long non-coding RNA (lncRNA, >200 nucleotides). ${ }^{3}$ Numerous studies have proved that miRNAs can post-transcriptionally repress translation or degrade mRNAs expression via binding to the $3^{\prime}$-untranslated region ( $3^{\prime}$-UTR) of the target gene. ${ }^{4}$

In addition, more and more research studies have shifted to lncRNA, which plays vital roles in various physiological and pathological processes. ${ }^{5}$ Recently, studies identified that lncRNA post-transcriptionally regulates the expression of $m$ RNA by competing for miRNA. ${ }^{38}$ Interference of dysregulated lncRNA and mRNAs (with complementary sequence of lncRNA) affected the expression level of target mRNAs through miRNAs, together harbored by lncRNAs, and target mRNAs called miRNA response elements (MRE). ${ }^{6,7}$ This miRNA-regulated lncRNA and mRNA network could be a part of the 
"competing endogenous RNA (ceRNA) hypothesis" proposed by Salmena et al. ${ }^{8}$ The ceRNA crosstalk represented that ceRNAs, as miRNA sponges, harbored the same MREs that can be negatively regulated by miRNAs, indicating that ceRNAs communicate with each other by competing for a pool of shared miRNAs. ${ }^{9}$ Experimental evidence validated that dysregulated expression of key lncRNAs could disturb the balance of ceRNA network, thus leading to the initialization and development of various cancers. ${ }^{10}$

Lung cancer remains the most common cause of cancer-related mortality worldwide, even with the decline in incidence and mortality rates. ${ }^{11}$ Non-small-cell lung cancer (NSCLC), of which the two common histologic subtypes are lung adenocarcinoma (LUAD) and lung squamous cell carcinoma (LUSC), accounts for $\sim 80 \%$ of lung cancer. ${ }^{12}$ It has been proved that genetic markers play a key role in the occurrence and development of NSCLC, such as singlenucleotide polymorphisms (SNPs), by dysregulating gene expression and molecular pathway. ${ }^{13-16}$ Therefore, further exploration of lncRNA might complement the physiological and pathological cellular processes.

Myocardial infarction-associated transcript (MIAT) was first identified as lncRNA in 2006. Researchers have previously performed case-control association studies: an SNP in MIAT was significantly associated with myocardial infarction (MI) in Japanese subjects, ${ }^{17}$ and two SNPs were related to paranoid schizophrenia in the Chinese Han population. ${ }^{18}$ MIAT might also act as ceRNA in some other diseases. ${ }^{19,20}$ However, whether MIAT is a part of these two molecular mechanisms in lung cancer development has not been well delineated.

With this in mind, we performed a case-control study of rs1061541 on IncRNA MIAT and dual luciferase reporter assay of interaction between MIAT and miR-548e-3p through rs 1061541, and then used bioinformatic methods and experiments to elucidate whether MIAT influences NSCLC by acting as ceRNA. This study focused on these two molecular mechanisms in lung cancer development.

\section{Patients and methods}

\section{Analysis of differentially expressed genes (DEGs) in The Cancer Genome Atlas (TCGA) database}

The RNA and miRNA expression data (level 3) and the corresponding clinical information of NSCLC patients were retrieved from TCGA database portal (https://tcga-data.nci. nih.gov/tcga/). Both RNA-seq data and miRNA-seq data of tumor tissues and adjacent non-tumor tissues were included, according to the histologic staging data. lncRNA and mRNA transcripts were annotated based on UCSC Genome Browser database (http://www.genome.ucsc.edu) database. After normalization of expression data, the DESeq package in $\mathrm{R}$ was used to identify the DEGs. Absolute fold change (including upregulation and downregulation) $>2$ and adjusted $P$-value $<$ 0.05 were set as cutoffs. Co-expression network and important functional modules were discovered by "Weighted Gene Co-expression Network Analysis” (WGCNA) package.

\section{Construction of ceRNA network}

Based on the ceRNA hypothesis, the ceRNA network was constructed by negative regulation between miRNAs and their target genes. DEG expression profiles were processed using Pearson Correlation analysis to measure significantly negative regulation between miRNA and 1 ncRNA or mRNA and positive correlation between lncRNA and mRNA $(r>0.8$ in MIAT-mRNAs and $r<-0.3$ in miRNA-MIAT/mRNA, $P<$ $0.05)$. In this study, IncRNA-miRNA and mRNA-miRNA interactions were determined according to mirCode (http:// www.mircode.org). The intersection of competing lncRNAmiRNA-mRNA was chosen from shared regulatory miRNAs. lncRNA and mRNA were coexpressional and predicted to be targets of miRNAs. The ceRNA network was visualized by Cytoscape v3.4.0.

\section{Patients and samples}

The samples in the case-control study included 1352 cases with NSCLC and 1320 controls from Shanghai Jiao Tong University School of Public Health and the Fourth Affiliated Hospital of China Medical University. Cases were histologically confirmed lung cancer without radio- or chemotherapy. Controls without evidence of any cancer were recruited from the same hospital during the same time, based on frequency matched to cases by gender and age ( \pm 5 years). This study was approved by the institutional review board of China Medical University, and signed written informed consent forms were provided by each subject.

\section{Genotyping}

Genomic DNA samples were extracted according to conventional standard phenol-chloroform extraction. Genotyping of MIAT rs1061541 was detected by the TaqMan SNP genotyping assay using the ABI 7500 FAST real-time polymerase chain reaction (PCR) system (Thermo Fisher Scientific, Waltham, MA, USA) with primer and probe (assay ID C_2467718_1) purchased from Thermo Fisher Scientific. To validate the results, $5 \%$ of random samples were retested, with a consistency rate of $100 \%$. 


\section{Cell lines and culture}

Human lung cancer cell lines, A549 and H1975, purchased from Shanghai Institute of Biochemistry and Cell Biology, Chinese Academy of Sciences (Shanghai, China), were cultured in RPMI-1640 medium with 10\% fetal bovine serum (Thermo Fisher Scientific), $100 \mathrm{U} / \mathrm{mL}$ penicillin, $100 \mathrm{mg} /$ $\mathrm{mL}$ streptomycin in air at $37^{\circ} \mathrm{C}$ with $5 \% \mathrm{CO}_{2}$.

\section{Dual luciferase reporter assay}

Transfection was performed in 24-well plates and cultured until attachment, then co-transfection of miR-548e-3p with MIAT-rs1061541-C or MIAT-rs 1061541-T mutant plasmids (GeneChem, Shanghai, China) in A549 cells was performed, respectively. Luciferase activities were measured by the Dual-Luciferase Reporter Assay System (Promega Corporation, Fitchburg, WI, USA) according to the manufacturer's protocol. Experimental results were normalized by Renilla luciferase activity for each transfected well and repeated three times.

\section{Real-time PCR (RT-PCR)}

A549 and H1975 cells were plated in six-well plates and transiently transfected with miR-133a-5p using PolyPlus reagent for $48 \mathrm{~h}$, respectively, according to the manufacturer's protocol. Total RNA was isolated using RNAiso Plus (KeyGEN BioTECH, Jiangsu, China) and reverse transcribed to cDNA using the PrimeScript RT reagent Kit with gDNA Eraser (Takara Bio, Shiga, Japan) following the manufacturer's instructions. GAPDH was used as internal reference. The RT-PCR of mRNA was performed using SYBR ${ }^{\circledR}$ Premix Ex Taq ${ }^{\mathrm{TM}}$ II (Takara). The RT-PCR of lncRNA was performed using TaqMan Gene Expression Master Mix and MIAT-specific TaqMan primers (assay ID: Hs00402814_m1) according to the manufacturer's protocol (Thermo Fisher Scientific).

\section{Statistical analysis}

In genotype analysis, the $\chi^{2}$ test and Student's $t$-test were used to examine difference between cases and controls in demographic variables. Logistic regression model was used to estimate the odds ratios (ORs) and their $95 \%$ confidence intervals (CIs). The rest of data were presented as mean \pm SD. Student's $t$-test (two-tailed) was used to compare two groups, and analysis of variance (ANOVA) with Bonferroni analysis was used in three groups. All these analyses were performed using Statistical Products and Services Solutions software (v.16.0, SPSS Inc., Chicago, IL, USA) unless otherwise specified. $P$-value $<0.05$ was considered statistically significant.

\section{Results}

We identified that 161 miRNAs, 107 lncRNAs and 4070 mRNAs were differentially expressed between LUAD tissues and adjacent non-tumor tissues; 308 miRNAs, 86 lncRNAs and 3959 mRNAs were in LUSC tissues and adjacent nontumor tissues (Figure 1). The expression of MIAT was found to be significantly increased in both histological subtypes.

\section{MIAT rs I06 I54I polymorphism in NSCLC}

We focused on SNP rs1061541 of lncRNA MIAT according to lncRNASNP (a database of functional SNPs in lncRNAs, http://bioinfo.life.hust.edu.cn/lncRNASNP/). The secondary structure of MIAT transcripts is changed by rs 1061541 polymorphisms and illustrated by the RNAfold program in Figure 2. Furthermore, SNP rs1061541 in lncRNA MIAT may create a binding site with miRNA-548e-3p and lead to a novel target site of miRNA-548e-3p and MIAT (Figure 3). To investigate whether MIAT rs1061541 polymorphisms influence the NSCLC, genotyping and Dual luciferase reporter assay were performed.

The samples in this study comprised 1352 cases and 1320 controls. Mean values of age of cases and controls (mean \pm SD) were $60.1 \pm 12.7$ and $59.2 \pm 15.3$ years, respectively. The difference was not statistically significant $(t=1.693$, $P=0.091)$. No significant difference was found in gender or smoking status between cases and controls ( $P$-values were 0.835 and 0.113 , respectively). The abovementioned results suggested that case and control groups were comparable in important characteristics.

Table 1 shows the genotype distribution of MIAT SNP rs1061541 in cases and controls, as well as its associations with lung cancer risk. The frequency of the rs $1061541 \mathrm{C}$ allele in the controls was 0.191 . The allele distributions were consistent with the Hardy-Weinberg equilibrium $\left(\chi^{2}=0.77\right.$ and $P=0.38$ ). For rs 1061541 in MIAT gene, carriers of the CC genotype revealed a lower risk of lung cancer compared with the homozygous wild genotype (adjusted $\mathrm{OR}=0.46,95 \% \mathrm{CI}=$ $0.29-0.74, P=0.001)$. Individuals carrying at least one $\mathrm{C}$ allele (CT/CC) were less likely to develop lung cancer (adjusted OR $=0.14,95 \% \mathrm{CI}=0.11-0.17, P<0.001)$. Further analyses were carried out by allele comparison, and the $C$ allele of rs 1061541 was found to associate with a decreased risk of lung cancer with an OR of $0.22(95 \% \mathrm{CI}=0.18-0.26, P<0.001)$. 
A
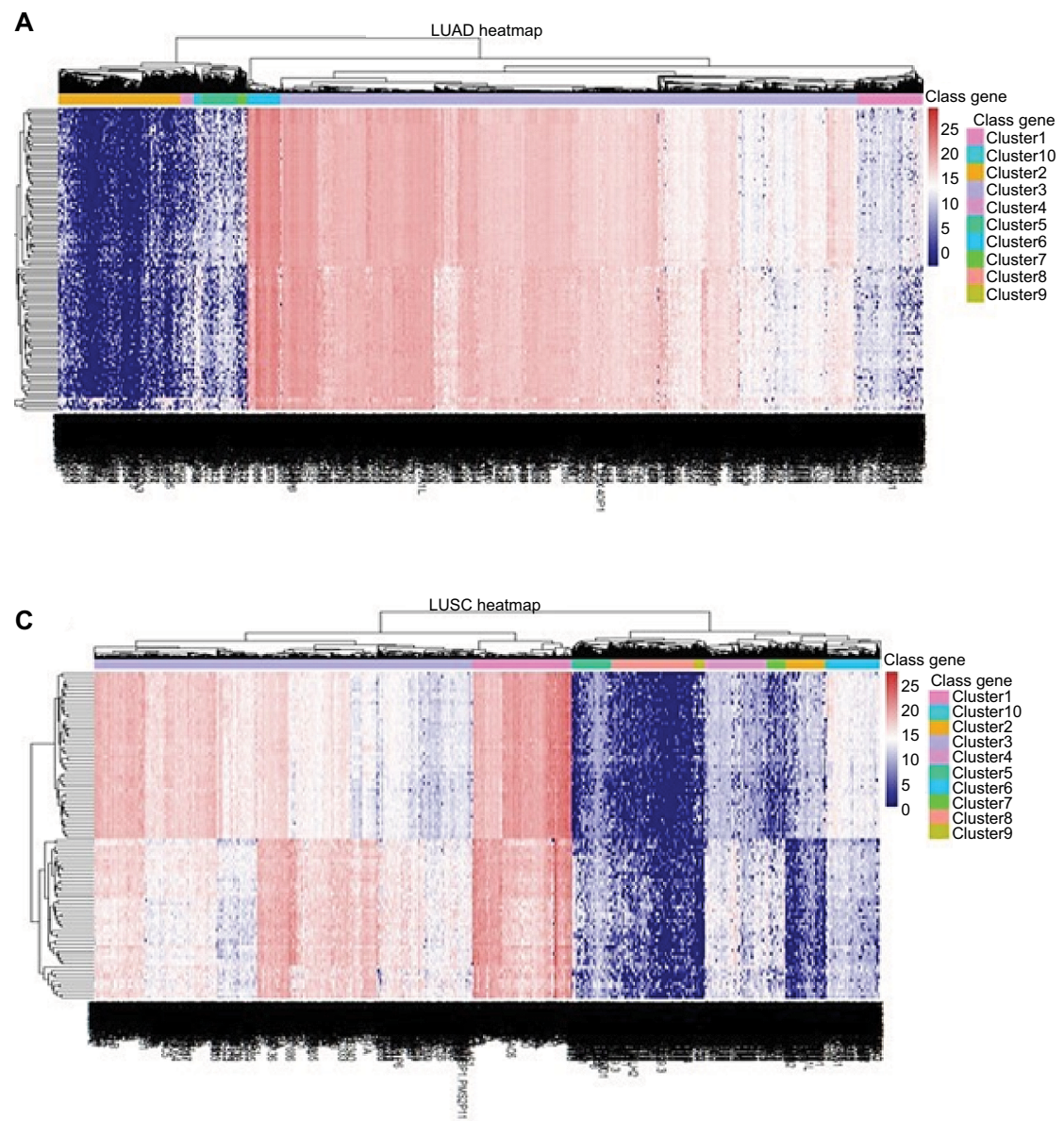

B

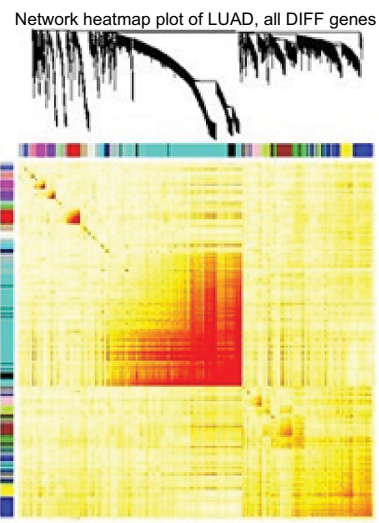

D
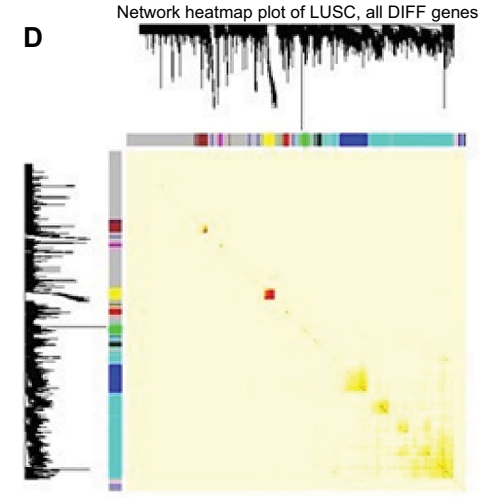

Figure I Heatmaps of differentially expressed genes in LUAD and LUSC.

Notes: Network visualization plots. (A and $\mathbf{C}$ ) The heatmap shows the differentially expressed genes (rows: low expression in blue and high in red) in LUAD and LUSC, respectively. (B and D) Visualizing expression pattern network using a heatmap plot by WGCNA (B for LUAD and D, for LUSC). The heatmap depicts the TOM among all differentially expressed genes in the analysis. Light color represents low overlap and progressively darker red color represents higher overlap. Genes that could not be assigned to a module are labeled gray.

Abbreviations: DIFF, differentially expressed; LUAD, lung adenocarcinoma; LUSC, lung squamous cell carcinoma; TOM, topological overlap matrix; WGCNA, Weighted Gene Co-expression Network Analysis.

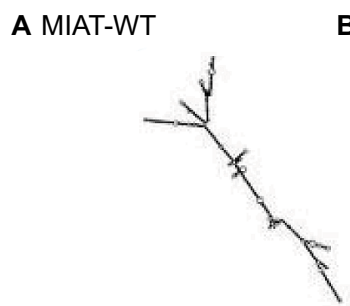

Figure 2 MIAT structure changes based on MIAT-WT (rs I06I54I-T) and MIATMUT (rsl06154I-C)

Notes: The secondary structure of MIAT transcripts of (A) MIAT-WT (rs I 06 I54I-T) and (B) MIAT-MUT (rsI06I54I-C).

Abbreviations: MIAT, myocardial infarction-associated transcript; MUT, mutation; WT, wild type.

Luciferase reporter assay was performed to further investigate whether rs 1061541 polymorphisms could influence the expression levels of MIAT by creating binding sites for miR$548 \mathrm{e}-3 \mathrm{p}$. It was predicted that such functional relevance and only one SNP rs1061541 existed in a potential binding seed in a public database. As shown in Figure 4, co-transfection of miR-548e-3p mimics with MIAT-rs1061541-T mutant plasmids resulted in significantly less luciferase activity; the same result was found in co-transfection of the miR-548e-3p mimics with the MIAT-rs 1061541-C. Additionally, no significant difference was observed between MIAT-rs1061541-C
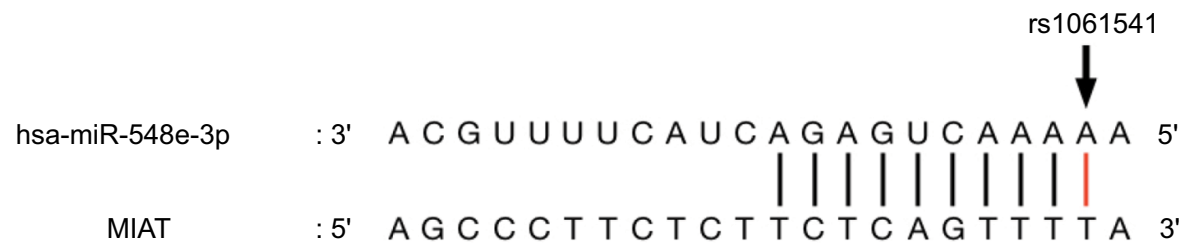

Figure 3 Predicted binding sequences of miR-548e-3p to MIAT, rs I06I54I shown in bold.

Abbreviation: MIAT, myocardial infarction-associated transcript. 
Table I Genotypes of the single-nucleotide polymorphisms rs I06/54I in lung cancer patients and control subjects and association with the risk of lung cancer

\begin{tabular}{|c|c|c|c|c|c|c|}
\hline Genotype & Cases, $\mathbf{n}$ & Controls, $n$ & OR $(95 \% \mathrm{Cl})$ & $P$ & Adjusted OR (95\% Cl) & Adjusted $P$ \\
\hline TT & 1245 & 860 & & & & \\
\hline CT & 74 & 417 & $0.12(0.09-0.16)$ & $<0.001$ & $0.10(0.08-0.14)$ & $<0.001$ \\
\hline $\mathrm{CC}$ & 33 & 43 & $0.53(0.33-0.84)$ & 0.007 & $0.46(0.29-0.74)$ & 0.001 \\
\hline $\mathrm{CT}+\mathrm{CC}$ & 107 & 460 & $0.16(0.13-0.20)$ & $<0.001$ & $0.14(0.11-0.17)$ & $<0.001$ \\
\hline $\mathrm{T}$ & 2564 & 2137 & & & & \\
\hline C & 140 & 503 & $0.23(0.19-0.28)$ & $<0.001$ & $0.22(0.18-0.26)$ & $<0.001$ \\
\hline
\end{tabular}

Note: Adjusted by age, gender and smoking status.

Abbreviations: $\mathrm{Cl}$, confidence interval; OR, odds ratio.

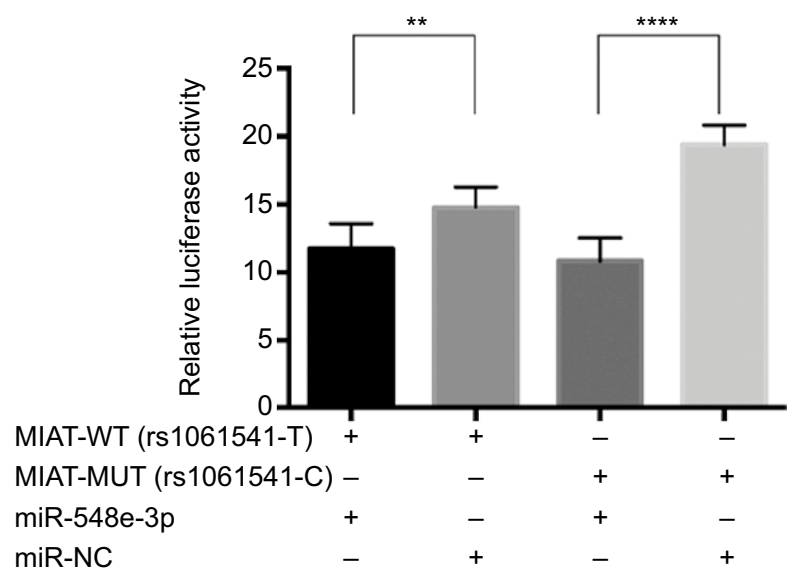

Figure 4 The results of luciferase reporter gene assay between miR-548e-3p and MIAT.

Notes: The effect of rsl06154I on MIAT interaction with miR-548e-3p. Relative reporter gene activity from constructs bearing an MIAT fragment with the rs 106I54I-T or rs 106154I-C allele in the A549 cell lines. "+" means co-transfected with MIAT-WT (rsI06I54I-T) or MIAT-MUT (rs I06I54I-C) with miRNA mimics in A549. Both MIAT rs 106I54I-T and rs 106I54I-C co-transfected with miR-548e$3 \mathrm{p}$ mimics showed less luciferase activity compared with NC controls, respectively. Renilla luciferase/firefly luciferase was calculated and normalized to NC controls as relative luciferase activity. Results are shown as mean \pm SD from three times repeated transfection experiments, each with six replicates. ${ }^{* *} P<0.01$; $* * * * P<0.0001$.

Abbreviations: NC, negative control; MIAT, myocardial infarction-associated transcript; MUT, mutation; WT, wild type.

and MIAT-rs1061541-T with miR-548e-3p mimics. These results revealed that luciferase activity was significantly reduced by cotransfecting both MIAT-rs1061541-C and MIAT-rs1061541-T constructs in the presence of miR$548 \mathrm{e}-3 \mathrm{p}$, indicating that MIAT is a target of miR-548e-3p.

\section{MIAT-centric ceRNA network}

Based on common ceRNA hypothesis, if MIAT absorbs miRNA X away from mRNA Y, the expression levels of MIAT and mRNA Y would be positively correlated; meanwhile, MIAT and mRNA Y would be negatively correlated with miRNA X. To further investigate whether MIAT participated in the development of NSCLC as ceRNA, we performed relevant comparison analysis integrating mRNA, IncRNA and miRNA expression profiles.

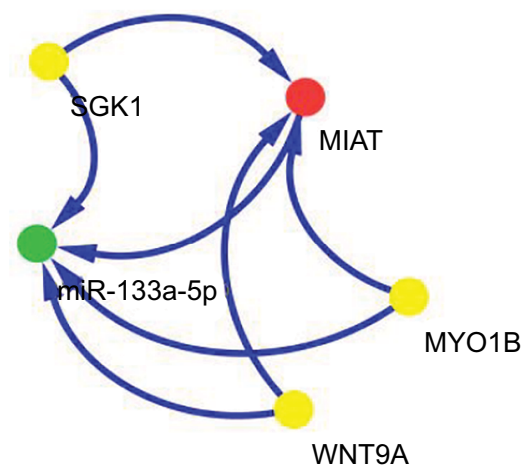

Figure 5 ceRNA network of MIAT in NSCLC.

Note: Red node represents IncRNA; yellow nodes represent mRNAs; and green node represents miRNA.

Abbreviations: ceRNA, competing endogenous RNA; IncRNA, long non-coding RNA; MIAT, myocardial infarction-associated transcript; MYOIB, myosin IB; NSCLC, non-small-cell lung cancer; SGKI, serum/glucocorticoid regulated kinase I; WNT9A, Wnt family member 9A.

A positive correlation was found between MIAT (red node) and myosin IB $(M Y O 1 B)$, serum/glucocorticoid regulated kinase 1 (SGK1) or Wnt family member 9A (WNT9A) (yellow nodes). The miR-133a-5p (green nodes) was negatively related to $M Y O 1 B, S G K 1, W N T 9 A$ or MIAT (Figure 5). Another remarkable signature was that these four RNAs were predicted by mirCode as potential target genes of miR-133a-5p. A dysregulated IncRNA-miRNA-mRNA ceRNA network could be constructed based on the abovementioned data.

Subsequently, to confirm the reliability and validity of the abovementioned ceRNA network, RT-PCR was performed. We found that overexpression of miR-133a-5p in A549 cells instead of $\mathrm{H} 1975$ cells (data not shown) depressed the expression of MYO1B, SGK1, WNT9A and MIAT (Figure 6). Similar results were also shown in RT-PCR and bioinformatics analysis, indicating that our ceRNA network is credible.

\section{Discussion}

With the development of microarray and sequencing, non-coding transcripts showed tissue-specific expression profiles. Although some lncRNAs are transcribed, this fact 
A

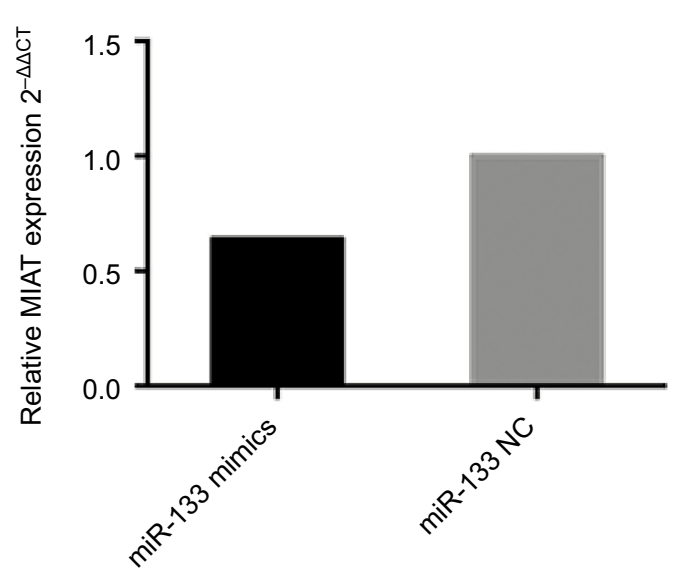

C

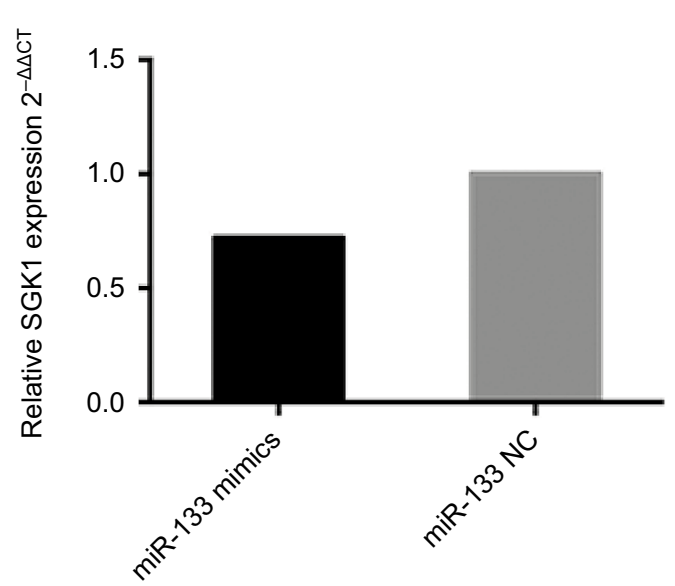

B

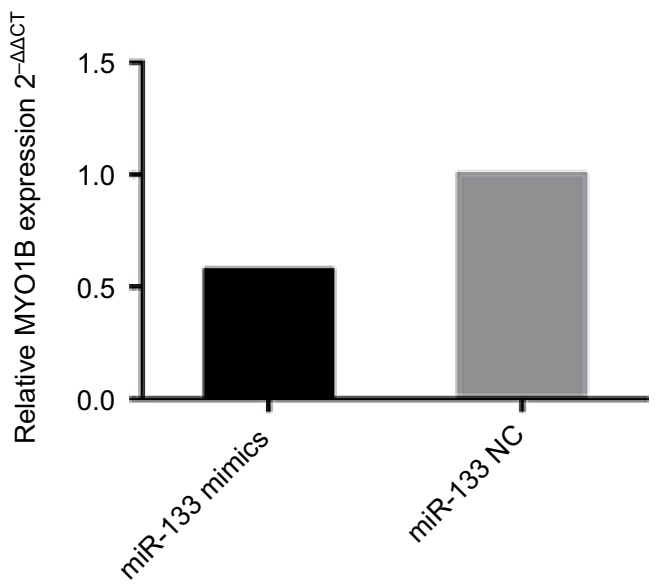

D

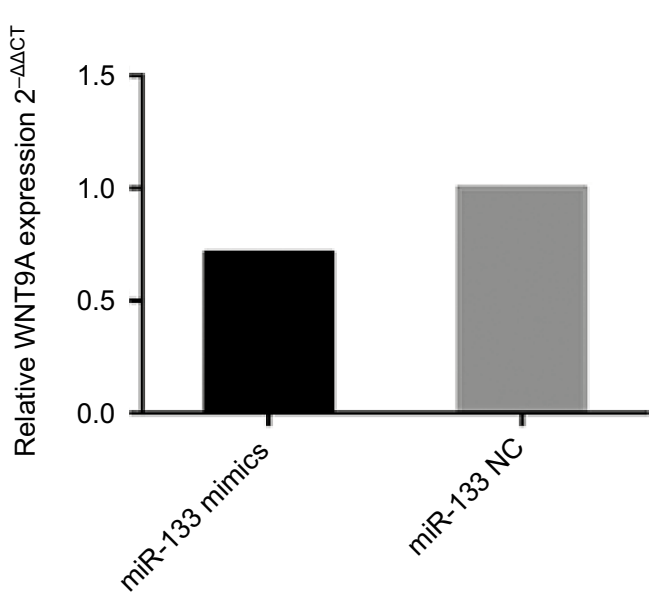

Figure 6 The expression levels of ceRNA in A549 following overexpression of miR-133 mimics compared to the negative control.

Notes: MIAT served as a ceRNA by binding miR-I33a-5p. The levels of (A) MIAT, (B) MYOIB, (C) SGKI and (D) WNT9A were significantly decreased, determined by RT-PCR in A549 cells transiently transfected with miR-133a-5p mimics. Results are represented as $2^{-\Delta \Delta C T}$ related to GAPDH levels from three experiments, each with six replicates. All $P$ values are from two-sided $t$-tests.

Abbreviations: ceRNA, competing endogenous RNA; MIAT, myocardial infarction-associated transcript; MYOIB, myosin IB; NC, negative control; NSCLC, non-small-cell lung cancer; RT-PCR, real-time polymerase chain reaction; SGKI, serum/glucocorticoid regulated kinase I; WNT9A, Wnt family member 9A.

does not necessarily imply that they perform crucial functions. However, a wealth of compelling evidence has proven that IncRNAs regulate gene expression through distinct transcriptional, post-transcriptional or epigenetic mechanisms. ${ }^{21}$ Recently, emerging evidence indicated that lncRNA transcripts showed biologically important functions, such as LUAD-associated transcript 1 (MALAT1), ${ }^{22,23}$ Homo sapiens TatD DNase domain containing 1 (TATDN1) ${ }^{24}$ and smoke and cancer-related long-chain ncRNA1 (SCAL1). ${ }^{25}$

MIAT, first identified as lncRNA a decade ago, was shown to affect endothelial cell functions ${ }^{17}$ and diabetic retinopathy. ${ }^{19}$ In this study, we focused on the lncRNA MIAT pathological processes in the development of NSCLC via two mechanisms.

First, we established a case-control study of the Chinese population to investigate SNPs on IncRNA MIAT in the development of NSCLC. Genotyping results showed that MIAT rs 1061541 in the CC genotype (adjusted OR $=0.46,95 \% \mathrm{CI}$ $=0.29-0.74, P=0.001)$ or the $\mathrm{CT}+\mathrm{CC}$ (adjusted $\mathrm{OR}=0.14$, $95 \% \mathrm{CI}=0.11-0.17, P<0.001)$ genotype acted as significantly protective factors compared with the TT genotype. Then, we found that miR-548e-3p could interact with MIAT-rs1061541$\mathrm{T}$ and MIAT-rs1061541-C; hence we demonstrated that there is no clear functional relationship between SNP rs1061541 and miR-548e-3p. Ishii et al reported an association of MIAT rs2301523 with MI patients in a large-scale Japanese population. ${ }^{17}$ Additionally, MIAT rs1894720 minor allele T carriers showed increased risk for paranoid schizophrenia in the Chinese Han population. ${ }^{18}$ Together with these findings, the importance of SNP in MIAT miRNAs may not yet be fully discovered.

Second, according to the TCGA project, our results demonstrated that MIAT harbors MRE interacted with 
miR-133a-5p, and miR-133a-5p targets $M Y O 1 B, S G K 1$ and $W N T 9 A$. To do this, we first identified that differentially expressed miRNAs were predicted to target both MIAT and DEGs. For each network, we analyzed the correlation between MIAT-targeted mRNAs, MIAT-miRNAs and mRNAs-miRNAs. The robustness and reliability of the ceRNA network were validated by experiments. The reason why only A549 cells showed the tendency, rather than H1975, may be the different genetic backgrounds between A549 (KRASmut) and H1975 (EGFRmut). The underlying mechanism is probably that Pao et $\mathrm{al}^{26}$ and Massarelli et al's $^{27}$ groups established which KRAS mutations are less sensitive to EGFR tyrosine kinase inhibitors (TKIs) than the mutations within EGFR. MIAT-associated ceRNA by binding miR-133a-5p might steadily activate the Ras-RafMEK-MAPK pathways through KRAS mutation, resulting in the resistance to treatment with EGFR TKIs. H1975 cells for the threonine at position 790 (T790M) mutation and leucine at position 858 (L858R) are drug-sensitive mutations in EGFR-TKIs, via activating multiple downstream signal transduction pathways to maintain survival and proliferation. ${ }^{28-30}$ Researchers previously found that MIAT functions as ceRNA competed for miR-150-5p with VEGF in central nervous system disorders ${ }^{31}$ and sponged the same miRNA to indirectly downregulate the expression of the zinc finger E-box binding homeobox 1 (ZEB1) in NSCLC. ${ }^{32}$ However, our analysis suggested that potential MIAT-centric ceRNA pathways via miR-133a-5p influence the occurrence and development of NSCLC.

Decreased expression of miR-133a-5p was reported in NSCLC patients. ${ }^{33,34}$ In this study, we indicated that miR133a-5p may play a key role in the MIAT-centric ceRNA network. Meanwhile, previous studies suggested that SGK1, the most represented member of the SGK family, showed high expression in NSCLC, ${ }^{35,36}$ and it was controlled by hormones (including gluco- and mineralocorticoids) and cellular stress (including cell shrinkage). ${ }^{37}$ Therefore, we represented an MIAT-miR-133a-5p-MYO1B $\backslash S G K 1 \backslash W N T 9 A$ transcription ceRNA network, which may be a potential compensatory mechanism in NSCLC by using combined bioinformatics methods and experimental approach. More comprehensive work about MIAT ceRNA network needs to be explored.

\section{Conclusion}

This study highlighted that SNPs of IncRNA MIAT have an impact on gene expression and the epigenetic machinery in lung cancer. Based on the first part of our findings, we can conclude that MIAT rs1061451 C-containing genotypes function as a protective factor with the development of NSCLC in Han Chinese populations. Moreover, we used bioinformatic methods and molecular cell biology analyses to characterize functions of the rs1061541, and the results showed that there was no influence of the polymorphisms of rs1061541 in MIAT interaction with miR-548e-3p. The second part of our findings suggested that MIAT, acting as ceRNA, took part in the expression of three mRNAs (MYO1B, SGK1 and $W N T 9 A$ ) by binding miR-133a-5p. These findings suggested that a disturbance between lncRNAs, miRNAs and mRNAs might lead to disease, including cancer.

\section{Acknowledgments}

This study was supported by grant no. 81502878 from the National Natural Science Foundation of China and no. 201501017 from the Doctoral Research Project of Liaoning. The authors appreciate the participants in this study.

\section{Disclosure}

The authors report no conflicts of interest in this work.

\section{References}

1. Djebali S, Davis CA, Merkel A, et al. Landscape of transcription in human cells. Nature. 2012;489(7414):101-108.

2. Huarte M. The emerging role of lncRNAs in cancer. Nat Med. 2015;21(11):1253-1261.

3. Derrien T, Johnson R, Bussotti G, et al. The GENCODE v7 catalog of human long noncoding RNAs: analysis of their gene structure, evolution, and expression. Genome Res. 2012;22(9):1775-1789.

4. Chan SH and Wang LH. Regulation of cancer metastasis by microRNAs. $J$ of Biomedical Science. 2015;22:9.

5. Huarte M. The emerging role of IncRNAs in cancer. Nat Med. 2015; 21:1253-1261

6. Wang D, Ding L, Wang L, et al. LncRNA MALAT1 enhances oncogenic activities of EZH2 in castration-resistant prostate cancer. Oncotarget. 2015;6(38):41045-41055.

7. Xie X, Tang B, Xiao YF, et al. Long non-coding RNAs in colorectal cancer. Oncotarget. 2016;7(5):5226-5239.

8. Salmena L, Poliseno L, Tay Y, Kats L, Pandolfi PP. A ceRNA hypothesis: the Rosetta Stone of a hidden RNA language? Cell. 2011;146(3):353-358

9. Yang C, Wu D, Gao L, et al. Competing endogenous RNA networks in human cancer: hypothesis, validation, and perspectives. Oncotarget. 2016;7(12):13479-13490.

10. Peng J, Zhang L, Yuan C, et al. Expression profile analysis of long noncoding RNA in ER-positive subtype breast cancer using microarray technique and bioinformatics. Cancer Manag Res. 2017;9:891-901.

11. Siegel RL, Miller KD, Jemal A. Cancer statistics, 2017. CA Cancer J Clin. 2017;67(1):7-30.

12. Lu C, Chen H, Shan Z, Yang L. Identification of differentially expressed genes between lung adenocarcinoma and lung squamous cell carcinoma by gene expression profiling. Mol Med Rep. 2016;14(2):1483-1490.

13. Wang SH, Zhang WJ, Wu XC, et al. Long non-coding RNA Malat1 promotes gallbladder cancer development by acting as a molecular sponge to regulate miR-206. Oncotarget. 2016;7(25):37857-37867.

14. Yin Z, Cui Z, Ren Y, Xia L, Li H, Zhou B. MiR-146a polymorphism correlates with lung cancer risk in Chinese nonsmoking females. Oncotarget. 2017;8(2):2275-2283. 
15. Lingzi X, Zhihua Y, Xuelian L, et al. Genetic variants in microRNAs predict non-small cell lung cancer prognosis in Chinese female population in a prospective cohort study. Oncotarget. 2016;7(50):83101-83114.

16. Agwa ES, Ma PC. Targeting the MET receptor tyrosine kinase in nonsmall cell lung cancer: emerging role of tivantinib. Cancer Manag Res. 2014;6:397-404.

17. Ishii N, Ozaki $\mathrm{K}$, Sato $\mathrm{H}$, et al. Identification of a novel non-coding RNA, MIAT, that confers risk of myocardial infarction. J Hum Genet. 2006;51(12):1087-1099.

18. Rao SQ, Hu HL, Ye N, Shen Y, Xu Q. Genetic variants in long noncoding RNA MIAT contribute to risk of paranoid schizophrenia in a Chinese Han population. Schizophr Res. 2015;166(1-3):125-130.

19. Yan B, Yao J, Liu JY, et al. IncRNA-MIAT regulates microvascular dysfunction by functioning as a competing endogenous RNA. Circ Res. 2015;116(7):1143-1156.

20. Zhu XH, Yuan YX, Rao SL, Wang P. LncRNA MIAT enhances cardiac hypertrophy partly through sponging miR-150. Eur Rev Med Pharmacol Sci. 2016;20(17):3653-3660.

21. Guttman M, Rinn JL. Modular regulatory principles of large non-coding RNAs. Nature. 2012;482(7385):339-346.

22. Gibb EA, Brown CJ, Lam WL. The functional role of long non-coding RNA in human carcinomas. Mol Cancer. 2011;10:38.

23. Ji P, Diederichs S, Wang W, et al. MALAT-1, a novel noncoding RNA, and thymosin beta4 predict metastasis and survival in early-stage nonsmall cell lung cancer. Oncogene. 2003;22(39):8031-8041.

24. Zequn N, Xuemei Z, Wei L, et al. The role and potential mechanisms of LncRNA-TATDN1 on metastasis and invasion of non-small cell lung cancer. Oncotarget. 2016;7(14):18219-18228.

25. Thai P, Statt S, Chen CH, Liang E, Campbell C, Wu R. Characterization of a novel long noncoding RNA, SCAL1, induced by cigarette smoke and elevated in lung cancer cell lines. Am J Respir Cell Mol Biol. 2013;49(2):204-211.

26. Pao W, Miller V, Zakowski M, et al. EGF receptor gene mutations are common in lung cancers from "never smokers" and are associated with sensitivity of tumors to gefitinib and erlotinib. Proc Natl Acad Sci USA. 2004;101(36):13306-13311.
27. Massarelli E, Varella-Garcia M, Tang X, et al. KRAS mutation is an important predictor of resistance to therapy with epidermal growth factor receptor tyrosine kinase inhibitors in non-small-cell lung cancer. Clin Cancer Res. 2007;13(10):2890-2896.

28. Pao W, Miller VA, Politi KA, et al. Acquired resistance of lung adenocarcinomas to gefitinib or erlotinib is associated with a second mutation in the EGFR kinase domain. PLoS Med. 2005;2(3):e73.

29. Balak MN, Gong Y, Riely GJ, et al. Novel D761Y and common secondary T790M mutations in epidermal growth factor receptor-mutant lung adenocarcinomas with acquired resistance to kinase inhibitors. Clin Cancer Res. 2006;12(21):6494-6501.

30. Inukai M, Toyooka S, Ito S, et al. Presence of epidermal growth factor receptor gene T790M mutation as a minor clone in non-small cell lung cancer. Cancer Res. 2006;66(16):7854-7858.

31. Jiang Q, Shan K, Qun-Wang X, et al. Long non-coding RNA-MIAT promotes neurovascular remodeling in the eye and brain. Oncotarget. 2016;7(31):49688-49698

32. Zhang HY, Zheng FS, Yang W, Lu JB. The long non-coding RNA MIAT regulates zinc finger E-box binding homeobox 1 expression by sponging miR-150 and promoting cell invasion in non-small-cell lung cancer. Gene. 2017;633:61-65.

33. Lan D, Zhang X, He R, et al. MiR-133a is downregulated in non-small cell lung cancer: a study of clinical significance. Eur JMed Res. 2015;20(1):50.

34. Wang Y, Li J, Chen H, et al. Down-regulation of miR-133a as a poor prognosticator in non-small cell lung cancer. Gene. 2016;591(2):333-337.

35. Abbruzzese C, Mattarocci S, Pizzuti L, et al. Determination of SGK1 mRNA in non-small cell lung cancer samples underlines high expression in squamous cell carcinomas. J Exp Clin Cancer Res. 2012;31:4.

36. Xiaobo Y, Qiang L, Xiong Q, et al. Serum and glucocorticoid kinase 1 promoted the growth and migration of non-small cell lung cancer cells. Gene. 2016;576(1 pt 2):339-346.

37. Lang F, Bohmer C, Palmada M, Seebohm G, Strutz-Seebohm N, Vallon V. (Patho)physiological significance of the serum- and glucocorticoidinducible kinase isoforms. Physiol Rev. 2006;86(4):1151-1178.

38. Tay Y, Rinn J, Pandolfi PP. The multilayered complexity of ceRNA crosstalk and competition. Nature. 2014;505(7483):344-352.
Cancer Management and Research

\section{Publish your work in this journal}

Cancer Management and Research is an international, peer-reviewed open access journal focusing on cancer research and the optimal use of preventative and integrated treatment interventions to achieve improved outcomes, enhanced survival and quality of life for the cancer patient. The manuscript management system is completely online and includes

\section{Dovepress}

a very quick and fair peer-review system, which is all easy to use. Visit http://www.dovepress.com/testimonials.php to read real quotes from published authors. 